\title{
1 First Glimpse of Gut Microbiota of Quarantine Insects in China
}

2

3 Yanxue $\mathrm{Yu}^{1 \#}$, Qi Wang ${ }^{2,3,4 \#}$, Ping $\mathrm{Zhou}^{1}, \mathrm{Na} \mathrm{Lv}^{3}$, Wei $\mathrm{Li}^{4,5,3}$, Fangqing Zhao ${ }^{6}$, Shuifang

$4 \mathrm{Zhu}^{1 *}$, and Di Liu ${ }^{4,5^{*}}$

5

6

9 100176, China;

102 Institute of Physical Science and information Technology, Anhui University, Hefei 230601,

11 China;

$12{ }^{3}$ CAS Key Laboratory of Pathogenic Microbiology and Immunology, Institute of

13 Microbiology, Chinese Academy of Sciences, Beijing 100101, China;

$14{ }^{4}$ Computational Virology Group, Center for Bacteria and Virus Resources and

15 Bioinformation, Wuhan Institute of Virology, Chinese Academy of Sciences, Wuhan 430071,

16 China;

175 University of Chinese Academy of Sciences, Beijing 100049, China.

$18{ }^{6}$ Computational Genomics Lab, Beijing Institutes of Life Science, Chinese Academy of

19 Sciences, Beijing 102206, China ;

$20 \quad{ }^{*}$ Contributed equally

$21 *$ Corresponding authors.

22 E-mail: liud@wh.iov.cn (DL), and zhusf@caiq.org.cn (SZ).

23 Running title: Yanxue Yu et al/Gut microbiota of quarantine insects

24 Total word count (from "Introduction" to "Materials and methods"): 3228

25 Total word count of article title: 10

26 Total word count of running title: 8

27 Total word count of key words: 8

28 Total word count of abstract: 176 
29 Total figures: 6

30 Total tables: 0

31 Total supplementary figures: 6

32 Total supplementary tables: 1

33 Total supplementary files: 7

34 Total number of references and number of references since $2014: 35 / 18$

35

36

37

38

39

40

41

42

43

44

45

46

47

48

49

50

51

52 
54 Abstract

55 Quarantine insects are economically important pests that frequently invade new

56 habitats. A rapid and accurate monitoring method to trace the geographical

57 source of invaders is therefore needed for prevention, detection, and eradication.

58 Current methods based on insect genetics are often too slow. We developed a

59 novel tracing method based on insect gut microbiota. The source location of

60 microbiota of insects can be used to rapidly determine the insects' geographic

61 origin. We analyzed 179 gut microbiota samples belonging to 591 individuals

62 of 22 quarantine insect species collected from 36 regions in China and abroad.

63 The gut microbiotas of these insects mainly included Actinobacteria,

64 Bacteroidetes, Cyanobacteria, Firmicutes, Proteobacteria, and Tenericutes. The

65 diversity of the insect gut microbiota was closely related to geographic and

66 environmental factors. Different insect species could be distinguished at the

67 phylum level of microbiota. Populations of individual insect species from

68 different regions could be distinguished at the genus level of microbiota. A

69 method for determining the geographical origin of invasive insect species was

70 tentatively established, but its practical applicability requires further study.

71 Keywords: Quarantine Insects; Geographical Origin; $16 \mathrm{~S}$ rDNA; Gut

72 Microbiota 


\section{Introduction}

75 Insects are common, diverse, and widely distributed throughout the world [1].

76 Quarantine insects are introduced species that may cause harm to agriculture,

77 forestry, stored products, or human health. Countries or regions must take

78 preventive and control measures to reduce the damage caused by quarantine

79 insects. Globally, quarantine insects cause annual losses of billions of dollars

80 [2,3], and China is seriously affected by many species of quarantine insects

81 [4,5]. There are 150 species or genera of quarantine insects on the List of

82 Imported Plant Quarantine Pests in the People's Republic of China

83 (http://www.aqsiq.gov.cn/xxgk_13386/zvfg/gfxwj/dzwjy/201706/t20170614_4

84 90858.htm). Some quarantine insects have a relatively limited distribution. For

85 example, Leptinotarsa decemlineata is mainly distributed in the northeast and

86 northwest of China, while other species such as Lissorhoptrus oryzophilus are

87 widely distributed in areas where its host plant (Oryza sativa) is grown.

88 Rapid and accurate methods are needed to identify geographical sources of

89 quarantine insects. This would aid in detection, monitoring, prevention, and

90 eradication. Current methods mainly use genetic tests such as DNA barcode

91 technology [6,7], restriction fragment length polymorphisms (RFLPs) [8],

92 single nucleotide polymorphisms (SNPs) [9], and microsatellite markers [10].

93 All of these methods are based on gene flow between populations, and they

94 require several generations of insects to complete. The genetic changes in

95 populations from different areas can only be used to infer the movement routes 
96 [5]. Currently, there are no methods that can trace the origin of an individual 97 insect within a short time interval.

Gut microbiota are often considered to be the second genome of insect

99 species. Gut microbiota are closely linked to the insect's environment; as such,

100 they are variable and can change rapidly. Microbe characteristics are related to

101 their area of origin; specific microbes can interact with quarantine insects

102 through the diet and thereby contribute to the insect gut microbiota. Gut

103 microbiota might thus provide clues to the geographical source of a quarantine

104 insect. The $16 \mathrm{~S}$ rDNA gene sequencing technique can be used for detection and

105 identification of insect gut microbiota [11]. In this study, we developed a

106 method to determine the geographical source of quarantine insects using gut

107 microbiota identified by $16 \mathrm{~S}$ rDNA gene sequencing.

108 Results

109 Sample collection and DNA extraction

110 A total of 591 quarantine insect individuals were collected. There were 22

111 species belonging to 19 genera and 13 families (Table S1). All insects were

112 analyzed using mitochondrial cytochromec oxidase subunit I (COI) genes for

113 species classification (Figure 1A). Photos and sizes of the species are shown in

114 Figure 1B. The 36 collection sites were located in eight provinces and covered

115 northeast, central, south, and northwest China (Figure 1, Table S1). Among the

116 quarantine insects studied, Ips typographus was widely distributed in China and

117 has been found in at least 20 provinces. The distributions of the other 21 
118 species varied, and each was only found in several provinces. In addition to the

119 insects collected in China, we also sampled 30 Ips typographus and five Cydia

120 pomonella individuals that originated outside of China and that were alive

121 when they were intercepted at the ports. In general, these insects are widely

122 distributed and established in China.

123 The tested insects varied greatly in size. To ensure that gene extraction was 124 not affected by insect size, all insect species were divided into three groups 125 according to their body length. If the body length of the adult insect was >5

$126 \mathrm{~mm}$, such as in Leptinotarsa decemlineata, Ips typographus, and Lymantria

127 dispar, one individual was used as one sample for gut microbiota analysis. If 128 the body length ranged from 2 to $5 \mathrm{~mm}$, such as in L. oryzophilus, C. 129 pomonella, and Bactrocera cucurbitae, five individuals collected from one site 130 were used as one sample for DNA extraction. If the body length was $<2 \mathrm{~mm}$, 131 such as in Trogoderma granarium, Eriosoma lanigerum, and Planococcus 132 minor, 10 insects collected from one site were used as one sample to extract 133 DNA. The purpose of several individuals making up a sample was to more 134 effectively extract DNA and also to reduce individual differences and human 135 impact. A total of 179 gut microbiota samples from quarantine insects were 136 obtained and subjected to $16 \mathrm{~S}$ rDNA sequencing (Table S1).

137 Sequencing and analysis of microbiota diversity

138 All 179 samples were simultaneously sequenced, producing a total of $13916,465,986$ reads. Each sample contained 50,000-150,000 reads and produced 
140 around 15-37 Mbp of data (Figure 2A). All data were submitted to the Genome

141 Sequence Archive (GSA), and the accession ID is CRA002386. After quality

142 control, there were 50,000-125,000 reads with 15-32 Mbp remaining for each

143 sample (Figure 2A). Clean data were then classified by the QIIME software

144 [12]. We classified 1527 taxa of microbes belonging to two kingdoms: Archaea

145 and Bacteria. There were 38 phyla, 98 classes, 178 orders, 277 families, and

146598 genera (Figure 2B). All 1527 taxa were identified to various taxonomic

147 levels. All 1527 could be identified to the kingdom level, 1525 to the phylum

148 level, 1505 to the class, 1450 to order, 1320 to family, 989 to genus, and 290 to

149 the species level (Figure 2B).

150 Due to the complexity of the data, we analyzed the composition of the

151 microbiota for each sample at the phylum level. Bacteroidetes, Firmicutes, and

152 Proteobacteria were the three dominant phyla in each sample. Actinobacteria

153 were common and ubiquitous (Figure 2C). Cyanobacteria and Tenericutes

154 were observed in some samples. The proportions of these phyla were different

155 in each sample due to the different host species and different collection sites.

156 Of the three dominant phyla, Proteobacteria had the maximum proportion,

157 followed by Firmicutes and Bacteroidetes (Figure 2D). Insect species whose

158 sample number was $>5$ were subjected to alpha diversity comparison. Except

159 for Eriosoma lanigerum, all other species exhibited no significant difference in

160 either Shannon, Simpson, PD_tree or the Chao1 index (Figure 2E). However,

161 the Shannon index and Simpson index of Eriosoma lanigerum (p. in Figure 2E) 
162 showed significant differences compared to the other insects except

163 Trogoderma granarium (e. in Figure 2E).

164 Screening of methods for determining geographical source based on 165 microbiota

166 To link the geographic source and microbiota of quarantine insects, we 167 analyzed microbiota data of all insects collected from the field using principal 168 component analysis (PCA). However, the dots representing insects from five 169 geographical areas were tangled, as shown in Figure 3A and Figure S1. In a 170 previous study, multiple factors were shown to affect the gut microbiota of 171 insects. These included insect species, developmental stage, diet, sex, and 172 geographical location [13-14]. According to the ADONIS test results based on 173 normalized abundance of gut microbiota, the effect size of geographical 174 factor $\left(\mathrm{R}^{2}=0.016\right)$ was a little higher than sex factor $\left(\mathrm{R}^{2}=0.007\right)$, but lower than 175 effect of developmental stage $\left(\mathrm{R}^{2}=0.028\right)$ and insect species $\left(\mathrm{R}^{2}=0.09\right)$. This 176 suggested that the geographical source of the insect was not the dominant factor 177 affecting the gut microbiota. Therefore, we used linear discriminant analysis 178 (LDA), a classification algorithm based on prior information. We compared 179 PCA and LDA by ADONIS test using reared insects whose geographical source 180 and diet factor were controlled. Given sex as prior information, $\operatorname{LDA}\left(\mathrm{R}^{2}=0.67\right.$, $181 \mathrm{P}$-value $=0.001)$ was better able to discriminate sex compared to $\mathrm{PCA}\left(\mathrm{R}^{2}=0.01\right.$, 182 P-value $=0.881)($ Figure 3B , Figures S2Aand S3A). For discriminate insects 183 species, a similar conclusion $\left(\mathrm{R}^{2}=0.71, \mathrm{P}\right.$-value $=0.001$ for $\mathrm{LDA}, \mathrm{R}^{2}=0.10$, 
$184 \mathrm{P}$-value $=0.36$ for $\mathrm{PCA}$ ) applied when species information was given to the

185 LDA (Figures S2B, and S3B). These results suggested that LDA has a better

186 diagnostic ability than PCA to extract specific factors affecting gut microbiota

187 (Figures 3E).

188 Verification of LDA's ability to distinguish geographical sourceTo

189 determine the ability of LDA to find the taxonomic level of microbes given 190 geographical information, we performed LDA at all taxonomic levels of 191 microbes except for kingdom. Insects from the same geographical area (marked 192 by dots in Figure 4A) were grouped together at the class, order, family, and 193 species levels. However, the insects from different geographical areas could not 194 be distinguished at the phylum or genus levels of the microbes (Figure $4 \mathbf{A}$ ).

195 The dots representing insects collected abroad were located far from the dots 196 representing insects collected in China. To determine the robustness of this 197 method, a Jackknife was performed 1000 times at all taxonomic levels of 198 microbes except for the kingdom level by dropping approximately $15 \%$ of the 199 sample each time. The distribution of accuracy from the jackknife method 200 showed that accuracy at the phylum level was lowest, while class level and 201 order level were higher than 0.95 (Figure 4B). Bootstrapping was also 202 performed 1000 times, and a similar result was obtained (Figure 4C). Class 203 and order were considered as proper taxonomic levels to distinguish 204 geographical clusters of host insects because of the high accuracy and low 205 standard deviation in both jackknife and bootstrap techniques (Figure $4 D$ and 
206 E).

207 LDA distinguishing insects from different geographical sourceTo study the 208 relationship between gut microbiota and host geographical source, the factor of 209 insect species was controlled, and LDA using geographical information was 210 performed using five insect species. These were Cydia pomonella, Ips 211 typographus, Leptinotarsa decemlineata, Lissorhoptrus oryzophilus, and 212 Henosepilachna vigintioctopuntata, each of which was sampled from at least 213 three areas. The Cydia pomonella result is shown in Figure 5, and the results 214 were similar for the other four selected insects (Figures S4 and S5). A total of 21518 Cydia pomonella were collected from four sites. Two sites, Dongning and 216 Mudanjiang, are in the northeast of China, while Urumqi and Korla are located 217 in the northwest. In Figure 5, using the ADONIS test (Figure S6 A and B), 218 those four sampling sites could be distinguished at most taxonomic levels of 219 microbes (P-value=0.04). There was not sufficient discrimination 220 (P-value=0.32) at the genus level when we analyzed the gut microbiota of 221 Henosepilachna vigintioctopuntata (Figures S5B and S6F), and discrimination 222 at the species level was inadequate $(\mathrm{P}$-value $=0.33)$ in the analysis of the 223 distribution of Lissorhoptrus oryzophilus (Figures S5A and S6E). Class level $224(\mathrm{P}$-value $=0.04)$, order level $(\mathrm{P}$-value=0.04), and family level $(\mathrm{P}$-value=0.06) 225 information were available, but the phylum level $(\mathrm{P}$-value=0.009) showed 226 better discriminatory ability (Figures S4, S5, and S6A-G). The phylum level 227 was the best for tracing a single insect species (Figure S6G). 
229 of gut microbiota. Cydia pomonella ( $\mathrm{P}-\mathrm{value}=0.01, \mathrm{R}^{2}=0.88$, Figure 6A, Figure

$230 \mathrm{~S} 6 \mathrm{H}$ ), Ips typographus (P-value $=0.004, \mathrm{R}^{2}=0.86$, Figure 6B, Figure S6I), and

231 Leptinotarsa decemlineata ( $\mathrm{P}$-value $=0.002, \mathrm{R}^{2}=0.86$, Figure 6C, Figure S6J)

232 could be traced accurately. For Lissorhoptrus oryzophilus, there was an overlap

233 between the Xundian group and the Menglian group (P-value $=0.002, \mathrm{R}^{2}=0.59$,

234 Figure 6D and Figure S6K). Both of these species were located in Yunnan

235 province. The Suifenhe group overlapped with the Hulin group for

236 Henosepilachna vigintioctopuntata ( $\mathrm{P}$-value $=0.001, \mathrm{R}^{2}=0.63$, Figure 6E)

237 (Figure S6L), though it is closer to Dongning in geography. A heat map was

238 used to find the features of the microbe phylum with the closest relation to the

239 host geographical source using relative abundance. A geographical correlation

240 was not obvious using one or several microbial phyla, but there was some

241 evidence for a pattern (Figure 6).

\section{Discussion}

243 To the best of our knowledge, this is the first attempt to trace quarantine insects

244 based on gut microbiota. There is no effective and fast method currently

245 available to identify the geographical source of quarantine insects. Although the

246 methods of DNA barcoding and RFLP can predict the geographical origin, it

247 takes many insect generations to detect gene flow from multiple origins $[15,16]$.

248 These methods are better at identifying the genetics of evolutionary

249 relationships or for working with species complexes [17,18]. 
250 Several methods were evaluated in this study. The method represented by

251 PCA may not be the most appropriate approach to extracting geographical

252 information. This is because species, sex, developmental stage, diet, and 253 geographical source of the host all affect the gut microbiota, while the 254 geographical source of the insects may not be the principal factor. We found 255 that methods based on prior information such as LDA were better for 256 distinguishing the geographical sources of insects using the gut microbiota. The 257 geographical factor was revealed by the composition patterns of gut microbes.

258 Supervised machine learning is effective at extracting composition patterns 259 from data using prior information to establish a prediction model. Therefore, 260 supervised learning is our recommended strategy. Some outliers were observed 261 in the LDA analysis because of the prior information. The sampling sites of 262 insects were defined as the geographical sources. However, the insects might 263 have originated in other places, and their gut microbiota may not have been 264 localized. This situation is inevitable in sampling, but an increased number of 265 samples and a robust algorithm may help to reduce this noise.

266 This study showed that different insect species can be distinguished using 267 the phylum level of gut microbiota. A single insect species from different 268 regions can be distinguished at the genus level of microbiota. For invasive 269 species in China with limited distributions such as $C$. pomonella, this method 270 can easily and quickly identify the source of the invasion. Similarly, for widely 271 distributed species like I. typographus, this method can distinguish specimens 
272 from China and abroad. This is a problem that cannot be addressed by genetic

273 tests or morphology. The insect gut microbiota is closely related to host states

274 of sex, diet, developmental stage, and niche occupation. For example,

275 honeybees living in different locations can differ significantly in their gut

276 microbiota. The structure of the microbial community can also differ among

277 bees depending on whether or not they are foraging on flowering rape crops

278 [19]. Worker honeybees and solitary bees also have different gut microbiota.

279 There are eight distinct bacterial species or phylotypes in worker honeybees,

280 three of which are Gram-positive species, for example Bifidobacterium, and

281 five Gram-negative species including $\beta$-proteobacterium [20]. In predatory

282 insects, the diversity of prey consumed can increase the diversity of bacteria in

283 the gut [21]. Meanwhile, the diversity of microorganisms is also greatly

284 influenced by differences in plants consumed [22-24]. The gut community of $L$.

285 decemlineata larvae feeding on tomato was dominated by the genera

286 Stenotrophomonas and Lactococcus, and larvae feeding on potato mainly had

287 Enterobacter [25].

288 The accuracy of $16 \mathrm{~S}$ amplifier sequencing was affected by the depth of

289 sequencing and the depth of coverage. The sequencing results showed that

290 differences in gut microbiota at the phylum level can distinguish insect species,

291 and differences at the genus level can distinguish the same insect species from

292 different source locations. It is feasible to trace the origin of insects from

293 different geographic sources using the genus level of gut microbes. However, 
294 due to the limited number of sequencing samples, the LDA values of some

295 samples did not cluster. These included C. pomonella collected from Ili and $L$.

296 decemlineata from Urumqi. However, for C. pomonella and I. typographus,

297 their native individuals and intercepted conspecifics were distinguishable in

298 this study.

299 This paper also addressed the problem of determining the gut microbiota in 300 small insects. The body length of different insect species varies greatly. Most 301 studies of insect gut microbes have dissected the gut and evaluated the 302 interaction between microbiota and host stage for bacteria identification 303 [26-30]. However, it is difficult to obtain individual guts from very small 304 insects. Some individuals represented one sample from which the genomic 305 DNA was extracted, and the microbiota was amplified from the entire insect 306 body. This approach may help solve the problem of individual differences and 307 small samples. The methods of genomic DNA extraction from insect gut 308 microbiota need further study to establish better standards for future research.

309 In summary, we developed a method to trace the origin of quarantine 310 insects using a prior information based LDA. Bacteroidetes, Firmicutes, and

311 Proteobacteria were the three dominant phyla in insect guts, and their relative 312 abundance differed among insect species. Class and order level of gut 313 microbiota can provide geographic information even though the gut microbiota

314 is masked by insect species differences. For a single insect species, the class, 315 order, and family levels of gut microbiota are useful taxonomic levels. 
316 Although this new method can determine the geographical source of many

317 insects without requiring species identification, its popularization and

318 application must be based on a database of insect gut microorganisms. If the

319 insect species can be identified, the geographical source of the insect might be

320 more accurately located. The quantity and quality of basic data will have a

321 large impact on identification accuracy, especially for prior information-based

322 methods. The gut microbiota of insects is complex and variable. A high-quality

323 background database could improve the accuracy and stability of the

324 identification model. To establish this database, multiple insect species should

325 be sampled, and the samples should be taken from different geographical areas.

326 To control the batch effects and ensure database quality, the sample processing

327 methods should be consistent.

328 Materials and methods

329 Sample preparation

330 Quarantine insects in China were used for this study. The collection sites were

331 determined based on previous monitoring records of quarantine insects. Most

332 insects were captured with a net. At each location, we collected at least 10

333 insect individuals for each species. Each insect was alive before it was

334 immersed into RNAlater® Stabilization Solution (Cat. No. AM7021, Ambion,

335 Austin, Texas, USA).

336 DNA extraction, 16S rRNA gene amplification and sequencing

337 We used three gut microbiota DNA extraction strategies based on the insect 
body length. Large individuals ( $>5 \mathrm{~mm}$ ) were extracted as one sample. Five intermediate size individuals $(2-5 \mathrm{~mm})$ or ten small individuals $(<5 \mathrm{~mm})$ were extracted as one sample. Before extraction, the surface of each insect was sterilized with $70 \%$ ethanol and washed twice with sterile PBS [31]. The insect abdomens or the whole insects were put into a special EP tube weighing $0.3 \mathrm{~g}$ along with $0.1 \mathrm{~mm}$ glass beads (Cat. No. 11079101, BioSpec, Oklahoma City, OK, USA). DNA extraction was done according to the QIAamp® Fast DNA Stool Mini Kit (Cat. No. 51604, Qiagen, Hilden, Germany). Samples were pretreated before the DNA extraction. Pretreatment steps were as follows: 1.4 $\mathrm{ml}$ of inhibit EX buffer was first added into the EP tube and the sample was ground in a bead beater for $1 \mathrm{~min}$. Second, the samples were incubated at $95^{\circ} \mathrm{C}$ for $10 \mathrm{~min}$, and then reground for $2 \mathrm{~min}$. The samples were then centrifuged for $1 \mathrm{~min}$ to pelletize the particles. The following steps were the same as those given in the kit protocol. The concentrations of DNA genes were measured with Nanodrop, using this as a template for PCR amplification.

The PCR reaction system used the protocol of HiFi HotStart DNA Polymerase (Cat. No. KR0369, Kapa Biosystems, Boston, MA, USA), and the experiments were carried out based on a two-step PCR reaction. The first PCR amplification was performed with the primer pairs under the following conditions: a denaturing step at $95^{\circ} \mathrm{C}$ for 5 min followed by 20 cycles of $98^{\circ} \mathrm{C}$ for $20 \mathrm{~s}, 52^{\circ} \mathrm{C}$ for $30 \mathrm{~s}, 72^{\circ} \mathrm{C}$ for $30 \mathrm{~s}$, and a final step of $5 \mathrm{~min}$ at $72^{\circ} \mathrm{C}$. The primer pairs were 5'-CCTACGGGNBGCASCAG-3' (forward) and 5'- 
360 GACTACNVGGGTATCTAATCC-3' (reverse). The PCR products were

361 purified with the kit from Agencourt AMPuer XPsystem (Cat. No. A63880,

362 Beckman Coulter, South San Francisco, CA, USA), and the purified products

363 were used with the second PCR amplification. The second PCR amplification

364 conditions were a denaturing step at $95^{\circ} \mathrm{C}$ for 5 min followed by 10 cycles of

$36598^{\circ} \mathrm{C}$ for $20 \mathrm{~s}, 60^{\circ} \mathrm{C}$ for $30 \mathrm{~s}, 72^{\circ} \mathrm{C}$ for $30 \mathrm{~s}$, and a final step of $5 \mathrm{~min}$ at $72^{\circ} \mathrm{C}$.

366 These primer pairs were the Illumina sequencing joint with different index,

367 having the V3 and V4 information of $16 \mathrm{~S}$. The PCR products were purified

368 using the same protocol as above. Then, the concentrations of each sample

369 were detected after electrophoresis. The method of sequencing was paired-end 250 bp (PE250) sequencing (HiSeq2500, Illumina, San Diego, CA, USA).

371 Quality control and taxonomy assignment

372 After sequencing, sequences were distributed into samples based on barcodes.

373 After we removed barcodes and primers, we trimmed $10 \mathrm{bp}$ at the start and end 374 of each read for quality control [32]. Sequences longer than 104 bp were 375 retained after trimming bases whose quality was below 20 using Sickle V1.33

376 software. Error correction was performed by SPAdes V3.1.9 software [33].

377 The workflow pick_open_reference_otus.py in QIIME v1.9.1 was used to pick 378 OTUs at $97 \%$ similarity and to assign a species level using the UCLUST 379 method in the GREEN GENE database.

380 Phylogenetics

381 We downloaded the COI gene sequences of the test insects from the Barcode of 
382 Life Data System (BOLD) database [34]. These 2374 sequences were aligned 383 using Clustal Omega (https://www.ebi.ac.uk/Tools/msa/clustalo/). Then, a 384 method based on maximum likelihood, RAxML-HPC2 v8.2.10 [35], was used 385 to construct a phylogenetic tree. We performed 1000 bootstrap replicates for 386 this tree after removing suspicious sequences. The tree was edited and $387 \quad$ visualized by Figtree v1.4.3.

\section{Data analysis and visualization}

389 The density distribution of sequencing quantity and composition of gut 390 microbiota were analyzed using $\mathrm{R}$ v3.4.1. The Alpha diversity index was 391 calculated using vegan package v2.5-3 in R. Principal component analysis 392 (PCA) was performed using $\mathrm{R}$ stats package v3.4.1. Linear discriminant 393 analysis (LDA) was performed using the MASS package in R software. The 394 heatmap was visualized using the R package pheatmap v1.0.10.

395 PCA for insects collected from five geographical areas was performed based on 396 relative abundance of microbes. LDA for all insects that were collected from 397 five geographical areas was performed based on relative abundance normalized 398 using the log function. LDA for reared insects and representative insects was 399 performed using relative abundance. The first and second components were 400 chosen for visualization using the $\mathrm{R}$ package ggplot2. The ratio of the LDA 401 classifying results to the original sample information was defined as the 402 accuracy. A Jackknife was performed 1000 times, excluding $15 \%$ of the sample 403 from each geographical source each time. Bootstrap resampling was performed 
4041000 times, and the number of bootstrap samples was equal to the original 405 samples. A heatmap was constructed using the relative abundance at the order 406 level using the $\mathrm{R}$ package pheatmap. Distance between each group in LDA or 407 PCA was measured with a permutational multivariate analysis of variance 408 (PERMANOVA) in the R package vegan v2.5-3. Comparison of ADONIS R2 409 and $\mathrm{P}$ value between LDA and PCA was performed with t-test by $\mathrm{R}$ package $410 \quad$ vegan $\mathrm{v}-2.5-3$.

\section{Availability}

412 All of the sequencing data have been deposited in Genome Sequence Archive 413 (GSA: CRA002386), and the data can be available freely at 414 https://bigd.big.ac.cn/search?dbId=gsa\&q=CRA002386.

\section{CRediT author statement}

416 Yanxue Yu: Resources, Data curation, Writing- Original draft preparation. Qi

417 Wang: Formal analysis, Writing- Original draft preparation. Ping Zhou:

418 Investigation. $\mathrm{Na}$ Lv:Methodology. Wei Li:Software. Fangqing Zhao:

419 Conceptualization, Methodology. Shuifang Zhu:Supervision. Di Liu:

420 Conceptualization, Writing-Review\&Editing.

\section{Competing interests}

422 The authors have declared no competing interests.

\section{Acknowledgments}

424 This work was supported by grants from the National Key Research and 425 Development Program of China (Grant Nos. 2016YFC1200800, 
427 language editing improved the wording of this report.

428 Authors' ORCID IDs

429 0000-0002-4582-8261(Yu Yanxue)

430 0000-0002-1728-8763(Wang Qi)

431 0000-0001-6553-6605(Zhou Ping)

432 0000-0003-0603-6170(Lv Na)

433 0000-0003-3364-8690(Li Wei)

$434 \quad$ 0000-0002-6216-1235(Zhao Fangqing)

435 0000-0002-3414-7698(Zhu Shuifang)

436 0000-0003-3693-2726(Liu Di)

$437 \quad$ References

438 [1] Basset Y, Cizek L, Cuenoud P. Arthropod diversity in a tropical forest. $439 \quad$ Science 2012;338:1481-4.

440 [2] Aukema JE, Leung B, Kovacs K, Chivers C, Britton KO, Englin J, et al. 441 Economic impacts of non-native forest insects in the continental United $442 \quad$ States. PLoS One 2011;6(9):e24587.

443 [3] Liebhold AM, Berec L, Brockerhoff EG, Epanchin-Niell RS, Yamanaka T. 444 Eradication of invading insect populations: from concepts to applications. $445 \quad$ Annu Rev Entomol 2015;61(1):335.

446 [4] Liu N, Li YC, Zhang RZ. Invasion of colorado potato beetle, Leptinotarsa 447 decemlineata, in China: dispersal, occurrence, and economic impact. 
Entomol Exp Appl 2012;143(3):201-217.

[5] Liu SS, De Barro PJ, Xu J, Luan JB, Zang LS, R YM, et al. Asymmetric mating interactions drive widespread invasion and displacement in a whitefly. Science 2007;318:1769-1772.

[6] Kress WJ, Garcia-Robledo C, Uriarte M, Erickson DL. DNA barcodes for ecology, evolution, and conservation. Trends Ecol Evol 2015;30(1):25-35.

[7] Smith AM, Rodriguez JJ, Whitfield JB, Hebert PDN. Extreme diversity of tropical parasitoid wasps exposed by iterative integration of natural history, DNA barcoding, morphology, and collections. Proc Natl Acad Sci U S A 2008;105(34):12359-64.

[8] Tan YD, Wan CL, Zhu YF, Lu C, Xiang ZH, Deng HW, et al. An amplified fragment length polymorphism map of the silkworm. Genetics 2001;157(3):1277-84.

[9] Lü ZC, Sun HB, Wan FH, Guo JY, Zhang GF. High variation in single nucleotide polymorphism (SNPs) and insertions/deletions (Indels) in the highly invasive Bemisia tabaci (Gennadius) (Hemiptera: Aleyrodidae) middle east-asia minor 1(MEAM1). Neotrop Entomol 2013;42:521-6.

[10] Tang XT, Tao HH, Du YZ. Microsatellite-based analysis of the genetic structure and diversity of Aleurocanthus spiniferus (Hemiptera: Aleyrodidae) from tea plants in China. Gene 2015;560(1):107-113.

[11] Knight R, Vrbanac A, Taylor BC, Aksenov A, Callewaert C, Debelius J, et 
al. Best practices for analysing microbiomes. Nat Rev Microbiol 2018; $16(7): 410-422$.

[12] Caporaso JG, Kuczynski J, Stombaugh J, Bittinger K, Bushman FD, Costello EK, et al. QIIME allows analysis of high-throughput community sequencing data. Nat Methods 2010;7(5):335-6.

[13] Yun JH, Roh SW, Whon TW, Jung MJ, Kim MS, Park DS, et al. Insect gut bacterial diversity determined by environmental habitat, diet, developmental stage, and phylogeny of host. Appl Environ Microbiol 2014;80(17):5254-5264.

[14] Kim JM, Choi MY, Kim JW, Lee SA, Ahn JH, Song J, et al. Effects of diet type, developmental stage, and gut compartment in the gut bacterial communities of two Cerambycidae species (Coleoptera). J Microbiol $2017 ; 55(1): 21-30$.

[15] Shi W, Kerdelhué C, Ye H. Genetic structure and colonization history of the fruit fly Bactrocera tau (Diptera: Tephritidae) in China and Southeast Asia. J Econ Entomol 2014;107(3):1256-1265.

[16] Wu ZZ, Li HM, Bin SY, Ma J, He HL, Li XF, et al. Sequence analysis of mitochondrial ND1 gene can reveal the genetic structure and origin of Bactrocera dorsalis s.s. BMC Evol Biol 2014;14(1):55.

[17] Clarke AR, Armstrong KF, Carmichael AE, Milne JR, Raghu S, Roderick $\mathrm{GK}$, et al. Invasive phytophagous pests arising through a recent tropical evolutionary raditation: the Bactrocera dorsalis complex of fruit flies. Annu 
492

493

494

495

496

497

498

499

500

501

502

503

504

505

506

507

508

509

510

511

512

513

Rev Entomol 2005;50(1): 293-319.

[18] Meeyen K, Nanork Sopaladawan P and Pramual P. Population structure, population history and DNA barcoding of fruit fly Bactrocera latifrons (Hendel) (Diptera: Tephritidae). Entomol Sci 2014;17(2):219-230.

[19] Jones JC, Fruciano C, Hildebrand F, Al Toufalilia H, Balfour NJ, et al. Gut microbiota composition is associated with environmental landscape in honey bees. Ecol Evol 2017;8(1):441-451.

[20] Engel P, Martinson VG, and Moran NA. Functional diversity within the simple gut microbiota of the honey bee. Proc Natl Acad Sci U S A 2012;109(27):11002-7.

[21] Tiede J, Scherber C, Mutschler J, Mcmahon K, Gratton C. Gut microbiomes of mobile predators vary with landscape contex and species identity. Ecol Evol 2017;7(20): 8545-57.

[22] Belda E, Pedrola L, Peretó J, Martínez-Blanch JF, Montagud A, Navarro E, et al. Microbial diversity in the midguts of field and lab-reared populations of the European corn borer Ostrinia nubilalis. PLoS One 2011;6(6):e21751.

[23] Priya NG, Ojha A, Kajla MK, Raj A \& Rajagopal R. Host plant induced variation in gut bacteria of Helicoverpa armigera. PLoS One 2012;7(1): e30768.

[24] Colman DR, Toolson EC \& Takacs-Vesbach CD. Do diet and taxonomy influence insect gut bacterial communities? Mol Ecol 2012;21:5124-5137. 
514 [25] Chung SH, Scully ED, Peiffer M, Geib SM, Rosa C, Hoover K \& Felton

515 GW. Host plant species determines symbiotic bacterial community

516 mediating suppression of plant defenses. Sci Rep 2017;(7):39690.

517 [26] Brune A and Dietrich C. The gut microbiota of termites: digesting the

[27] Engel $\mathrm{P}$ and Moran NA. The gut microbiota of insects-diversity in structure and function. FEMS Microbiol Rev 2013;37:699-735.

522 [28] Dietrich C, Köhler T, Brune A. The cockroach origin of the termite gut

[29] Dillon RJ, Webster G, Weightman AJ, Charnley AK. Diversity of gut microbiota increases with aging and starvation in the desert locust. Antonie van Leeuwenhoek 2010;97:69-77.

[30] Xu LT, Deng JD, Zhou FY, Cheng CH, Zhang LW, et al. Gut microbiota in an invasive bark beetle infected by a pathogenic fungus accelerates beetle mortality. J Pest Sci 2018;1-9.

[31] Pang XJ, Xiao XP, Liu Y, Zhang RD, Liu JY, et al. Mosquito C-type lectins maintain gut microbiome homeostasis. Nat Microbiol 1 2016;16023: 1-24.

534 [32] Schirmer M, Ijaz UZ, D'Amore R, Hall N, Sloan WT, Quince C, et al. 535 Insight into biases and sequencing errors for amplicon sequencing with the 
$536 \quad$ Illumina MiSeq platform. Nucleic Acids Res 2015;43(6):e37.

537 [33] Bankevich A, Nurk S, Antipov D, Gurevich AA, Dvorkin M, Kulikov AS,

$538 \quad$ et al. SPAdes: a new genome assembly algorithm and its applications to

$539 \quad$ single-cell sequencing. J Comput Biol 2012;19(5):455-77.

540 [34] Ratnasingham S, Hebert PD. bold: The barcode of life data system. Mol

$541 \quad$ Ecol Notes 2007;7(3):355-364.

542 [35] Stamatakis A. RAxML Version 8: A tool for phylogenetic analysis and post-analysis of large phylogenies.

Bioinformatics

$544 \quad 2014 ; 30(9): 1312-1313$. 


\section{$546 \quad$ Figure legends}

\section{Figure 1 Collection of insect samples and analysis of evolutionary relationship}

548 A. Phylogenetic tree of insects using the COI gene. Each color represents a different species.

549 The lower-case letters represent species that correspond to those in the photo and map below.

550 B. Photos of sampled insects. C. Insect sampling sites. The sampling sites are indicated on

551 the map of China, and the four main collection areas are marked with four different colors. $\mathbf{J}$

552 is Carpomya vesuviana Costa.

\section{Figure 2 Density distribution of sequencing quantity}

554 A. The left panel is the data size in base pairs (bp) per sample $(\mathrm{M}=$ million). The right panel 555 is the sequence number per sample. B. The left panel is the number of different taxa 556 identified at each taxonomic level. The right panel is the number of microbes that could be 557 identified at each taxonomic level $(\mathrm{k}=$ kingdom, $\mathrm{p}=$ phylum, $\mathrm{c}=$ class, $\mathrm{o}=$ order, $\mathrm{f}=$ family, $558 \mathrm{~g}=$ genus, $\mathrm{s}=$ species). C. Compositions of gut microbiota for each sample at the phylum 559 level. D. Relative abundance of three dominant phyla in each insect species. The color of the 560 box corresponds to that in C. E. Alpha diversity of eight representative insect species (**, P $561<0.01 . *, \mathrm{P}<0.05)$.

562 Figure 3 Screening of methods for determining geographical source based on insects' 563 microbiotaA. PCA method of distinguishing insects collected from five geographical areas. 564 Result of principal component analysis (PCA) showed that dots representing insects from 565 five geographical regions were intertwined and difficult to distinguish. B. LDA methods of 566 distinguishing insects considering the sex factor. Result of linear discriminant analysis (LDA) 567 showed that dots can gather into clusters, which can distinguish insects of different sexes. C. 568 LDA methods of distinguishing insects considering the insect species factor.These dots can 569 gather into clusters, which can distinguish insects of different species.. D. Effect size of each 570 insect related factor. (R2 was calculated from ADONIS test)

571 E. Comparison of ADONIS effect size between LDA and PCA using t-test. (**, $\mathrm{P}<0.01 ; * * *$, $572 \quad \mathrm{P}<0.001)$

573 Figure 4 Verification of LDA's ability to distinguish all insects at each microbial 574 taxonomic level 
575 A. The points in the linear discriminant analysis (LDA) were shaped and circled by sampling

576 the geographic area. The transparent circle delineates several insects that were intercepted at

577 entry ports. The dots representing insects collected abroad were located far from the dots

578 representing insects collected in China. Class and order were considered as proper

579 taxonomic levels to distinguish geographical clusters of host insects. B. Distribution of

580 accuracy at each taxonomic level of gut microbes via the jackknife. C. Distribution of

581 accuracy at each taxonomic level of gut microbes via the bootstrap. D., E. Mean and SD of

582 accuracy for the jackknife and bootstrap. The high accuracy and low standard deviation were

583 verified with the jackknife and bootstrap.

584 Figure 5 Application of LDA method in each taxonomic level of gut microbes of Cydia

585 pomonella.A total of 18 Cydia pomonella were collected from four sites. Two sites,

586 Dongning (red dots) and Mudanjiang (green dots), are in the northeast of China, while

587 Urumqi (blue dots) and Korla (purple dots) are located in the northwest. Dots of the same

588 color were distributed in clusters, showing that four sampling sites could be distinguished at 589 most taxonomic levels of microbes.

590 Figure 6 LDA and heatmap for gut microbes of five representative insects. Heatmap

591 showing relative abundance of each insect at the phylum level. The bar on top of the 592 heatmap represents the geographical source of the insect. Each of the five insects was 593 analyzed at the phylum level of gut microbiota. Cydia pomonella (A), Ips typographus (B), 594 and Leptinotarsa decemlineata (C) could be traced accurately. For Lissorhoptrus oryzophilus 595 (D), there was an overlap between the Xundian group and the Menglian group. Both of these 596 species were located in Yunnan province. The Suifenhe group overlapped with the Hulin 597 group for Henosepilachna vigintioctopuntata (E), though it is closer to Dongning in 598 geography.

599

600 Supplementary material

601 Table S1 Detailed information of insect samples

602 Figure S1 Distinguishing ability of PCA for tracing the geographical source of insects

603 Each point represents an insect, and the point color represents the geographical area where 
604 the insect was collected. Hollow points represent the imported insects.

605 Figure S2 LDA for discriminating sex or species of insects based on gut microbes.

606 The linear discriminant analysis (LDA) can distinguish insects with sex (A) and species 607 differences (B).Figure S3 Discrimination comparison of LDA and PCA using the 608 PERMANOVA test

609

610 The methods of principal component analysis (PCA, left panel) and linear discriminant 611 analysis (LDA, right panel) were compared by ADONIS test using reared insects whose 612 geographical source and diet factor were controlled. A. Comparison of two methods in sex 613 factors. LDA was better able to discriminate sex compared to PCA. B. Comparison of two 614 methods in species factors. For discriminate insects species, $\mathrm{R}^{2}=0.71, \mathrm{p}$-value $=0.001$ for 615 LDA, $\mathrm{R}^{2}=0.10, \mathrm{p}$-value $=0.36$ for PCA. These results suggested that LDA has a better 616 diagnostic ability than PCA to extract specific factors affecting gut microbiota.

Figure S4 Verification of LDA's ability to distinguish Ips typographus and Leptinotarsa decemlineata at each microbial taxonomic level

A. The linear discriminant analysis (LDA) distinguished Ips typographus at all taxonomic level. A point represented an insect, the point color showed its geographical source, and the same geographical area was clustered together. Red areas indicated samples from abroad, green areas indicated samples from Suifenhe in Heilongiiang Province, and blue areas indicated samples from Taicang in Jiangsu Province. B. The linear discriminant analysis (LDA) distinguished Leptinotarsa decemlineata at all taxonomic level. A point represents represented an insect, and the point color shows showed its geographical source, and the same geographical area was clustered together. Red areas indicated samples from Urumqi, green areas and blue areas respectively indicated samples from Mishan and Suifenhe in Heilongjiang Province..

\section{Figure S5 Verification of LDA's ability to distinguish Lissorhoptrus oryzophilus and} Henosepilachna vigintioctopuntata at each microbial taxonomic level. A point represents an insect, and the point color shows its geographical source. A. The linear discriminant analysis (LDA) distinguished Lissorhoptrus oryzophilus at all taxonomic level. A point represented an insect, the point color showed its geographical source, and the same 
635 geographical area was clustered together. Red areas indicated samples from Dongning in

636 Heilongjiang Province, green areas indicated samples from Menglian, and blue areas

637 indicated samples from Songming, and purple areas indicated samples from Xundian in

638 Yunnan Province. B. The linear discriminant analysis (LDA) distinguished

639 Henosepilachna vigintioctopuntata at all taxonomic level. A point represents represented an

640 insect, and the point color shows showed its geographical source, and the same geographical

641 area was clustered together. Red areas indicated samples from Dongning, green areas and

642 blue areas respectively indicated samples from Hulin and Suifenhe in Heilongjiang Province.

\section{Figure S6 PERMANOVA test for five insect species}

$645 \mathrm{P}$ values of PERMANOVA tests at each taxonomic level of gut microbiota for Cydia 646 pomonella (A, B), Ips typographus (C), Leptinotarsa decemlineata (D), Lissorhoptrus 647 oryzophilus $(\mathbf{E})$, and Henosepilachna vigintioctopuntata $(\mathbf{F})$ G. The $\mathrm{P}$ values of all five 648 insect species. PERMANOVA test of distance between each group in LDA for Cydia 649 pomonella (H), Ips typographus (I), Leptinotarsa decemlineata (J), Lissorhoptrus 650 oryzophilus $(\mathbf{K})$, and Henosepilachna vigintioctopuntata $(\mathbf{L})$. 


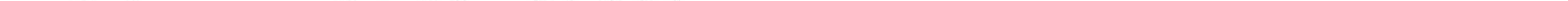



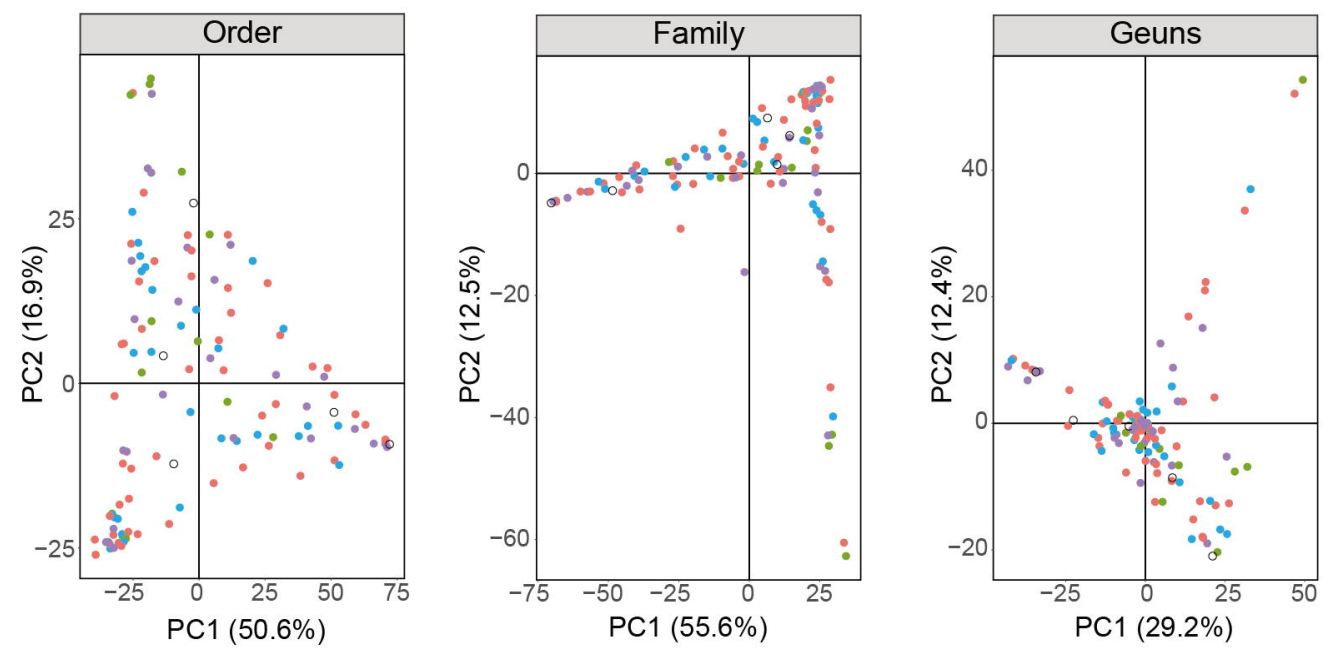

Point color

B.

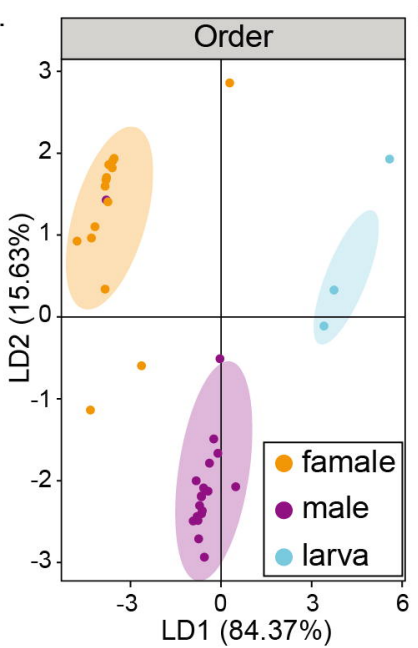

C.

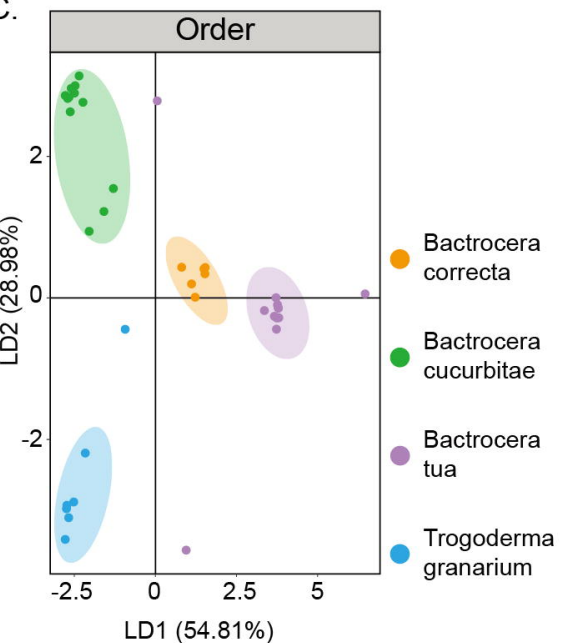

D.

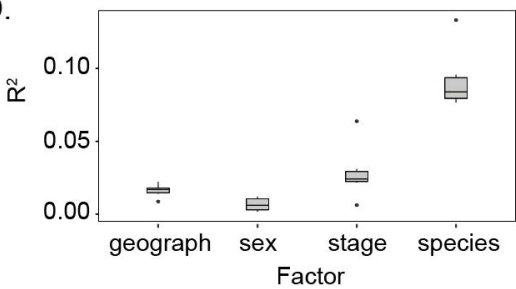

E.

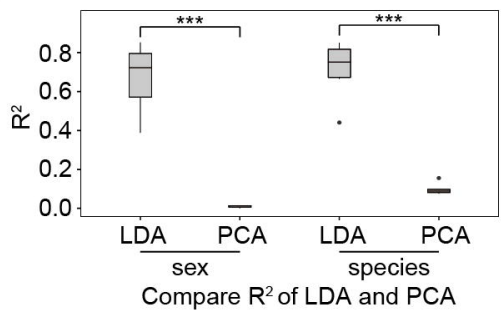



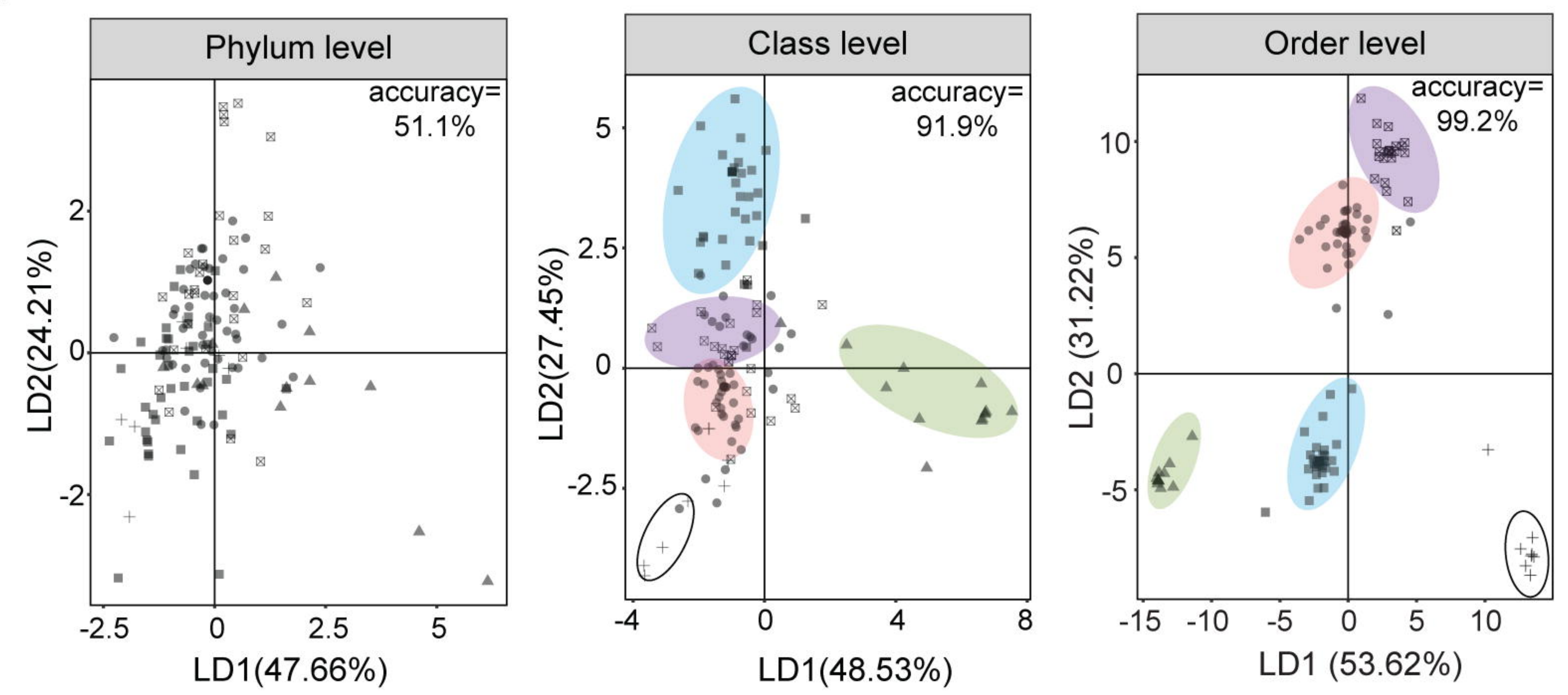

Location

- North East

- Central

- South

+ Import

凶 North West
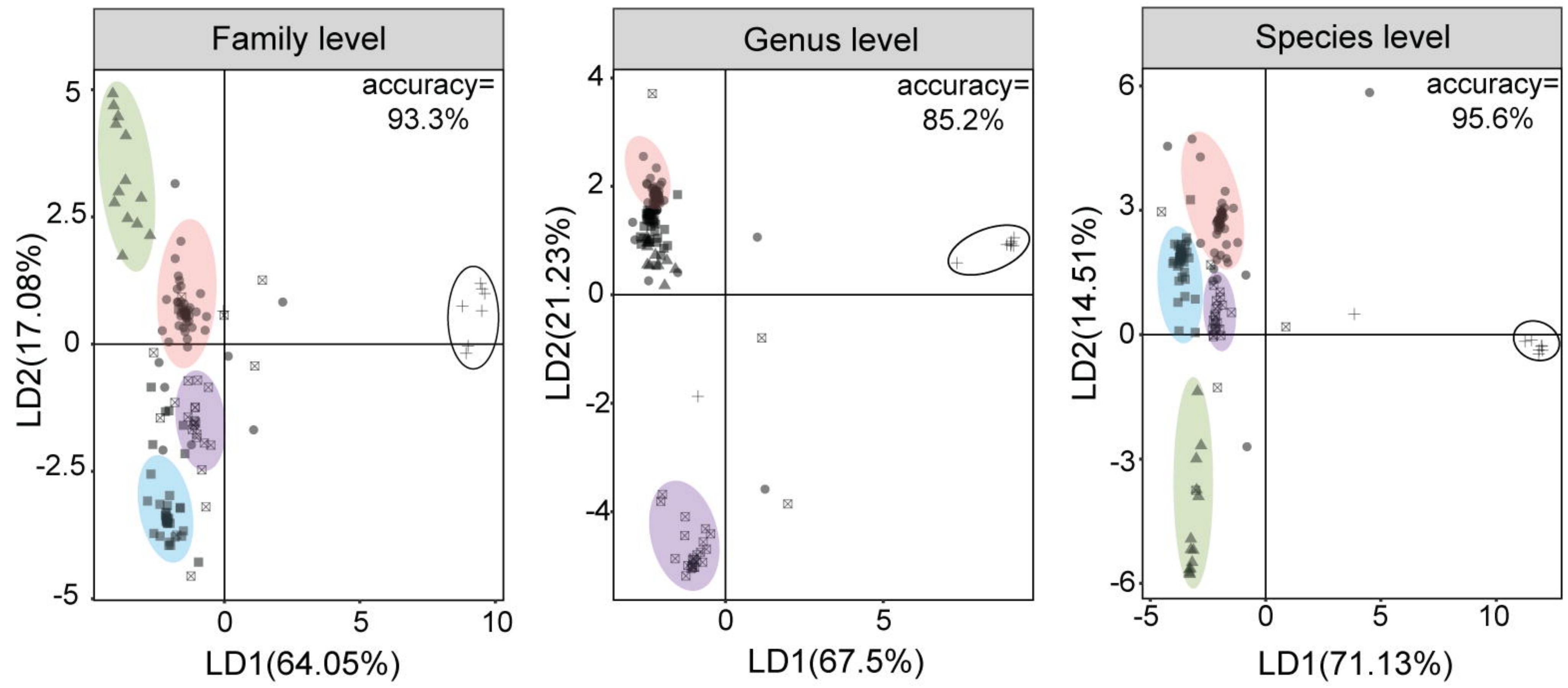

Circle color

B.
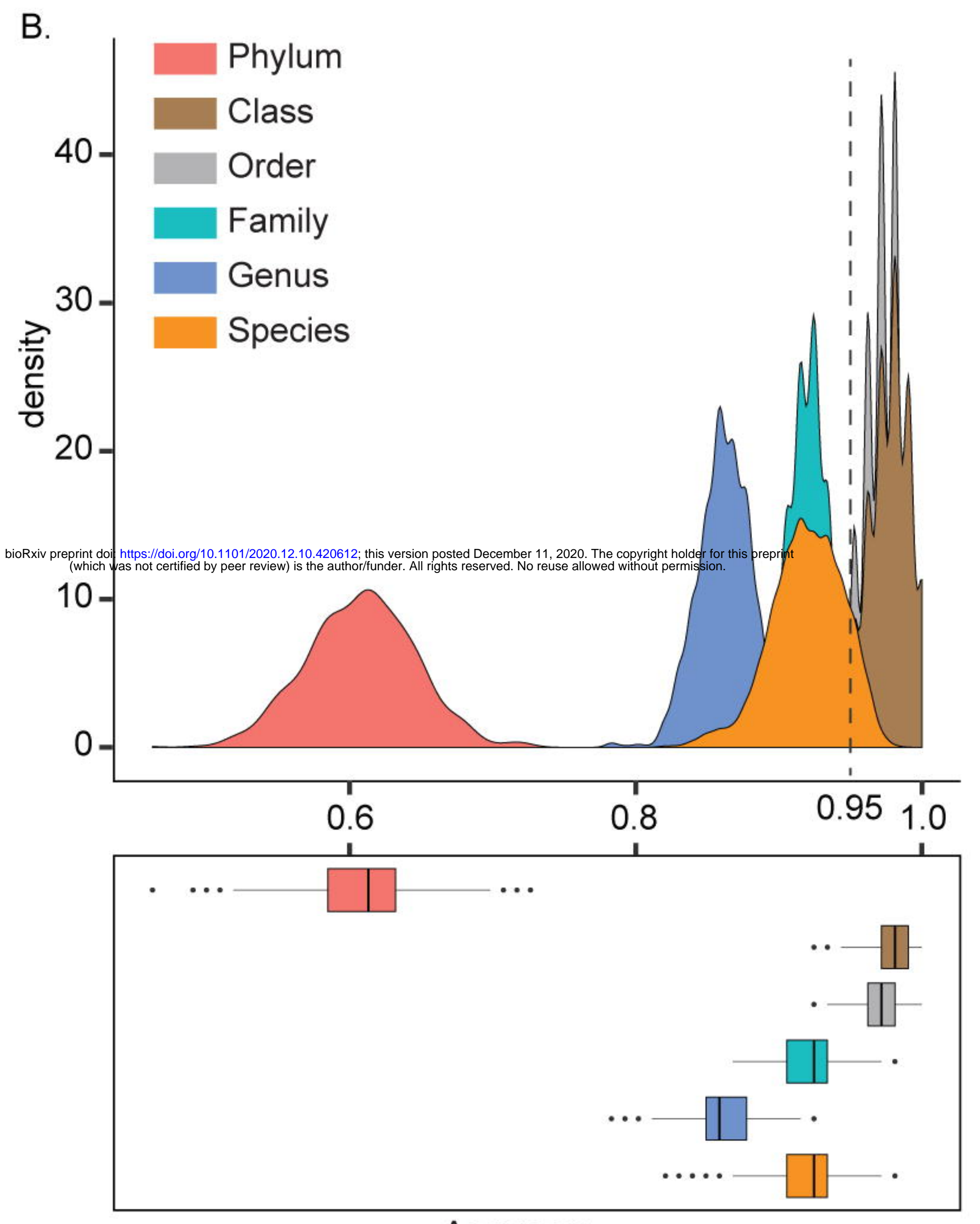

D.

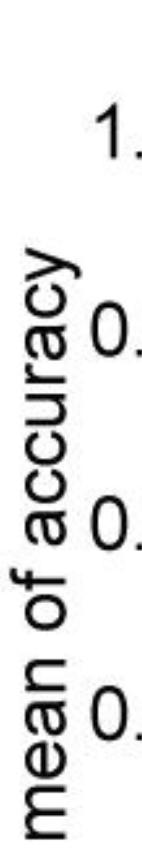
0.00

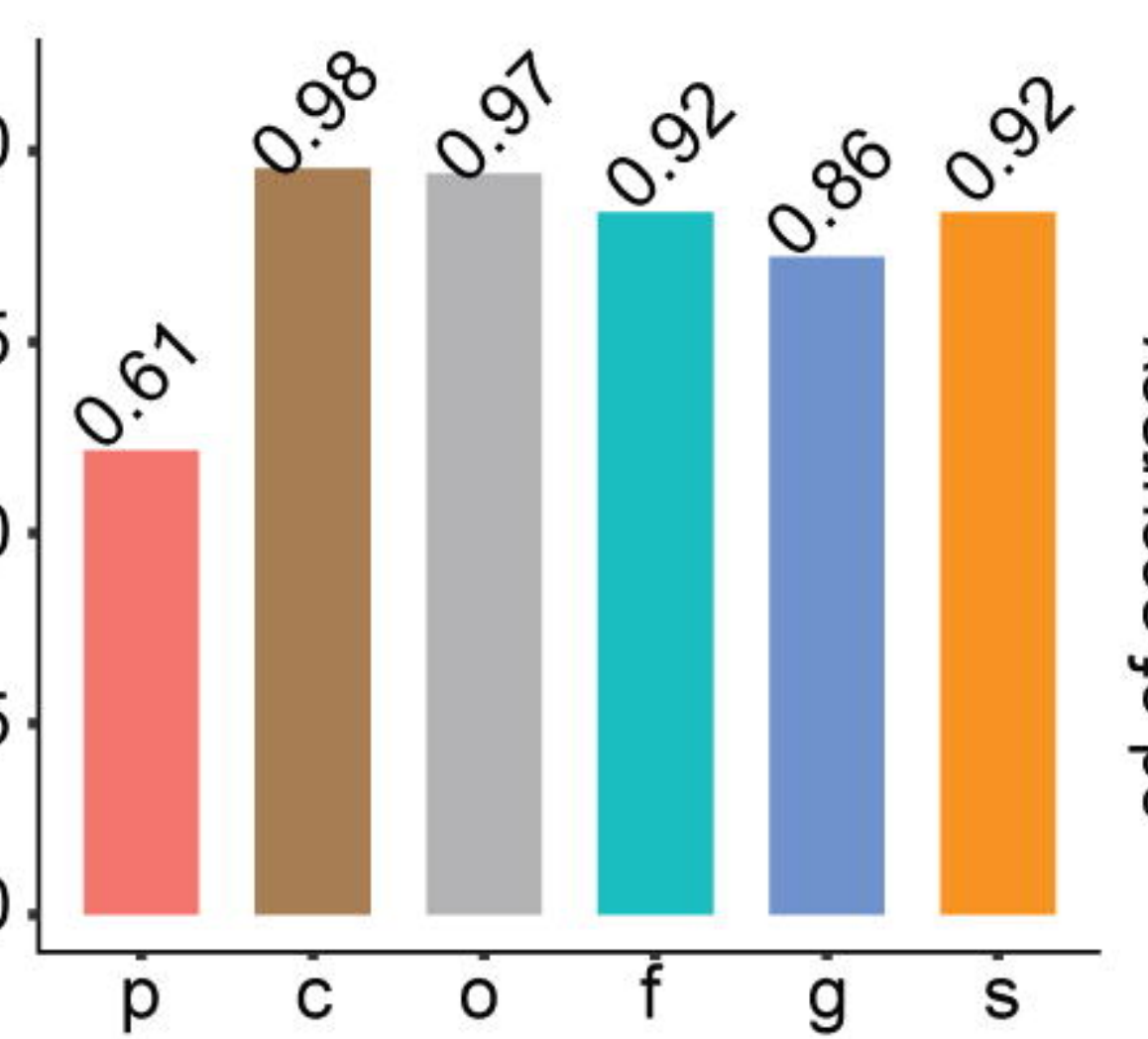
Taxonomy level
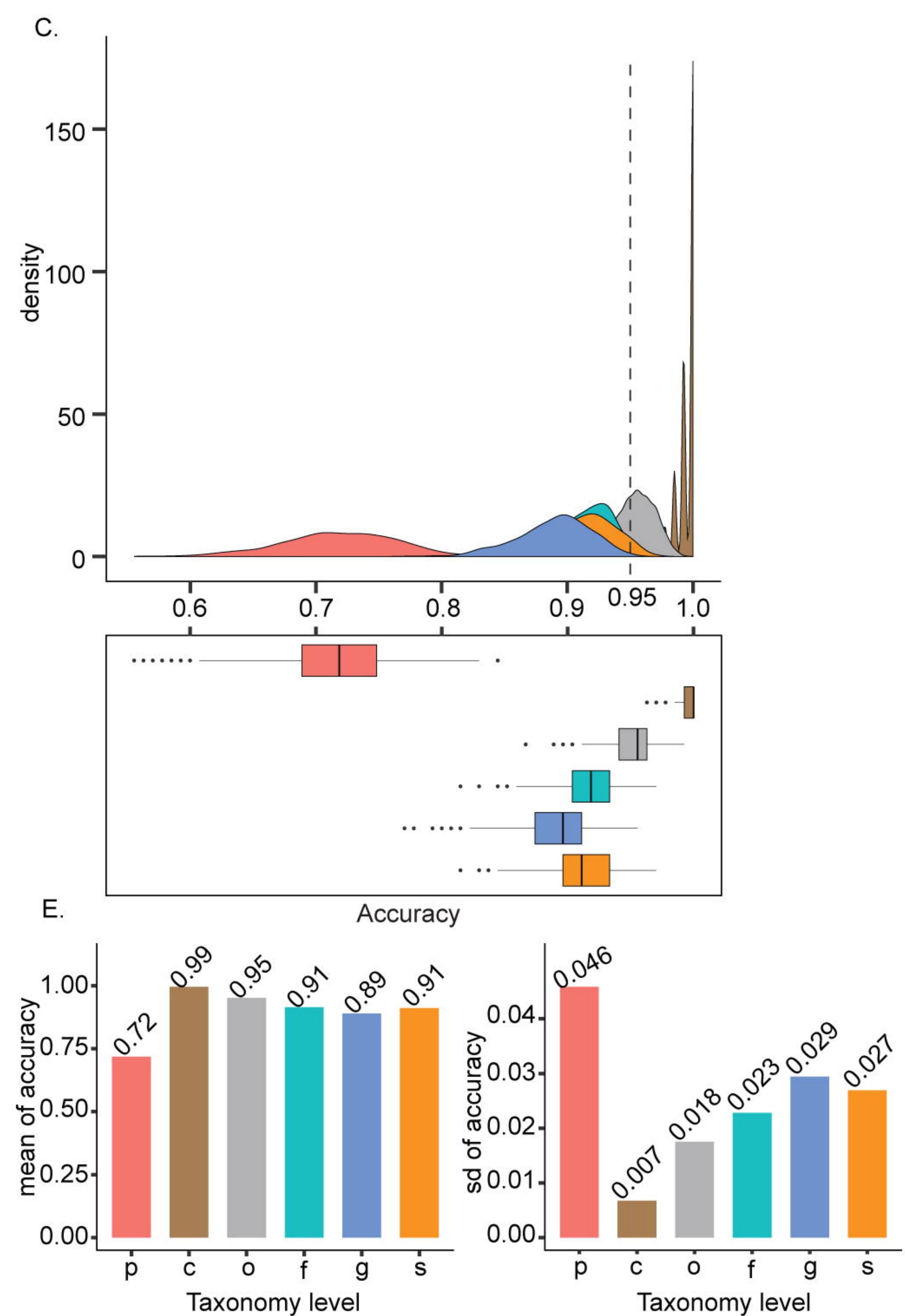


\section{Phylum}
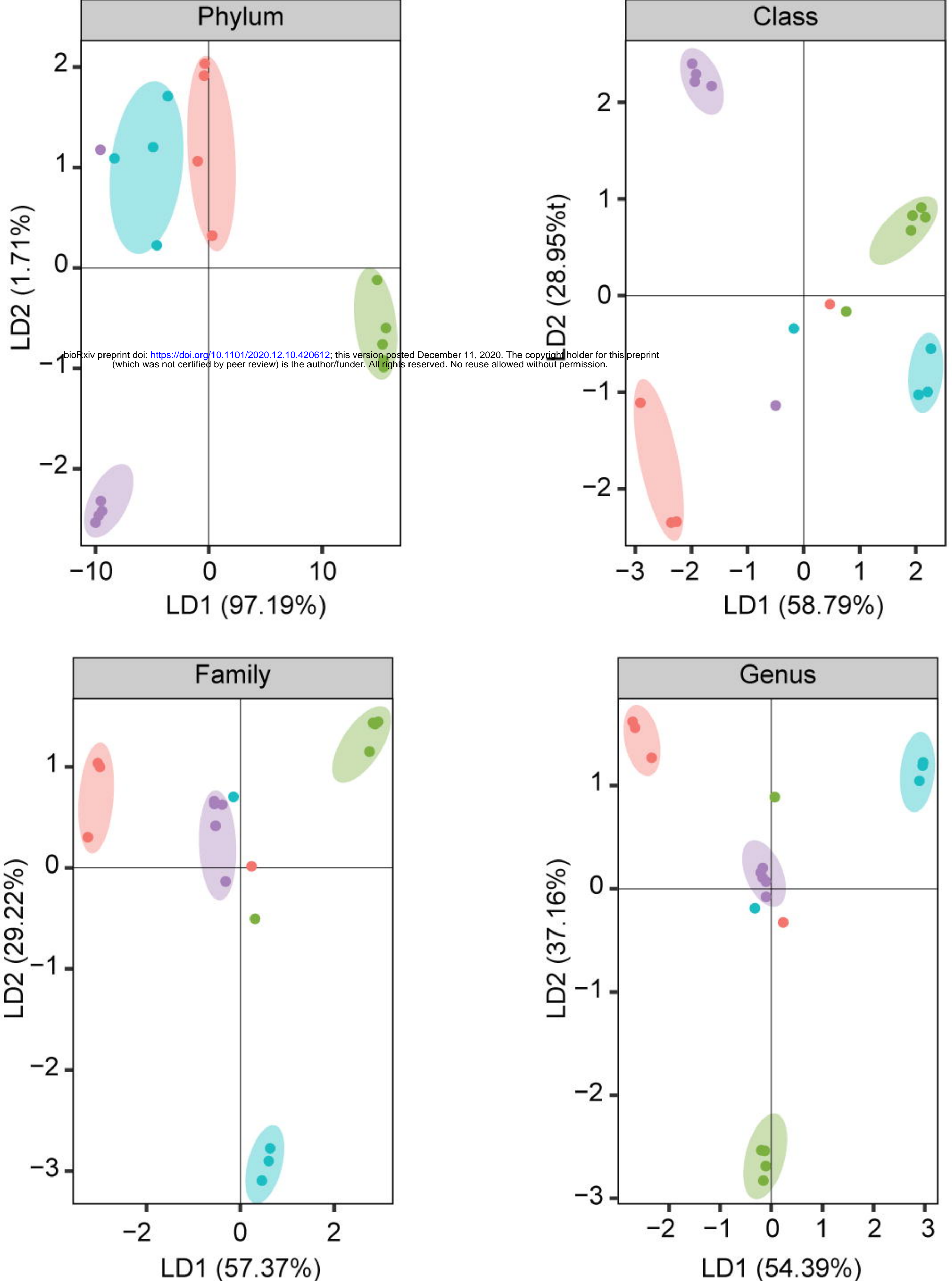

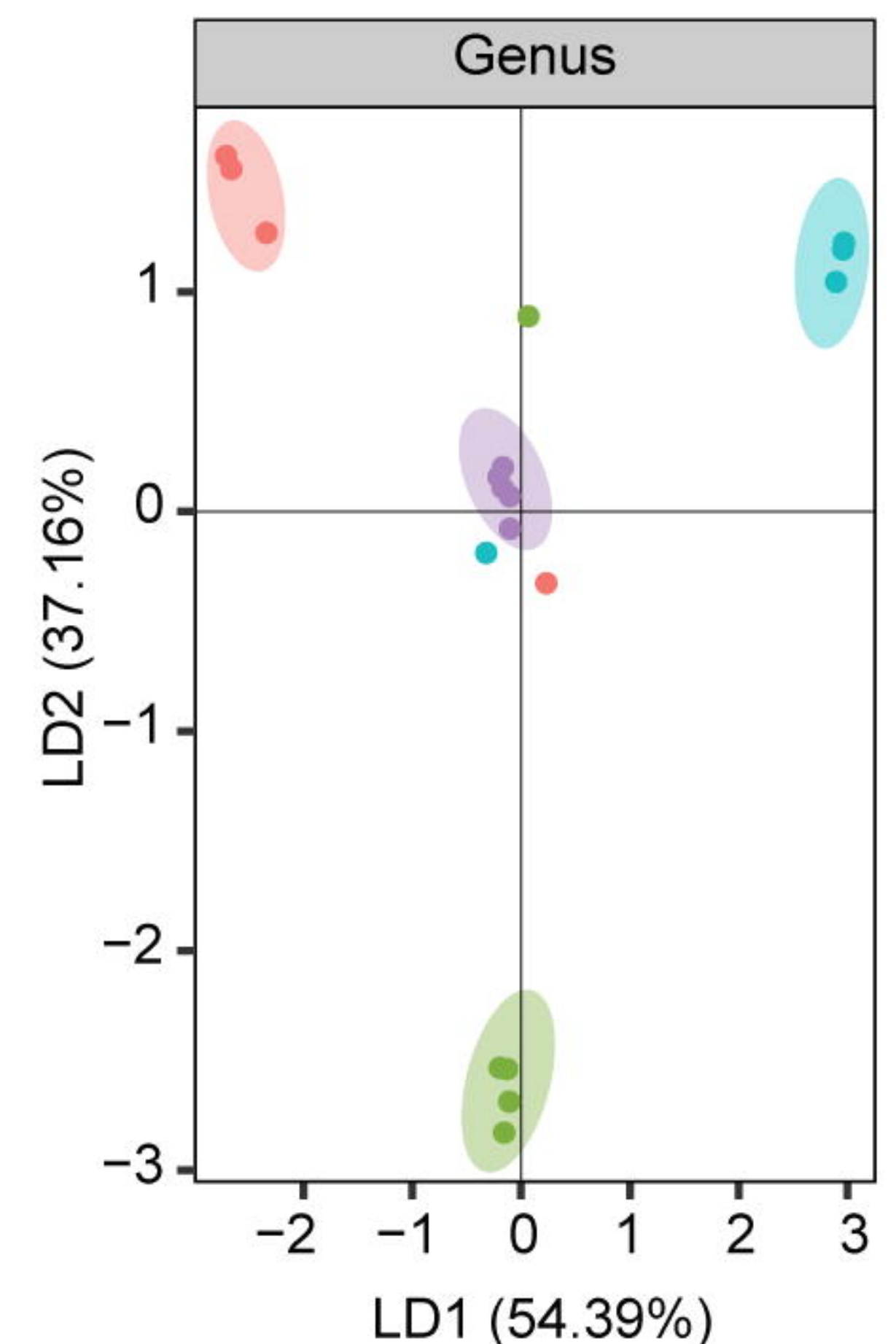

Cydia pomonella

Location

- Dongning

- Mudanjiang

- Urumqi

Korla

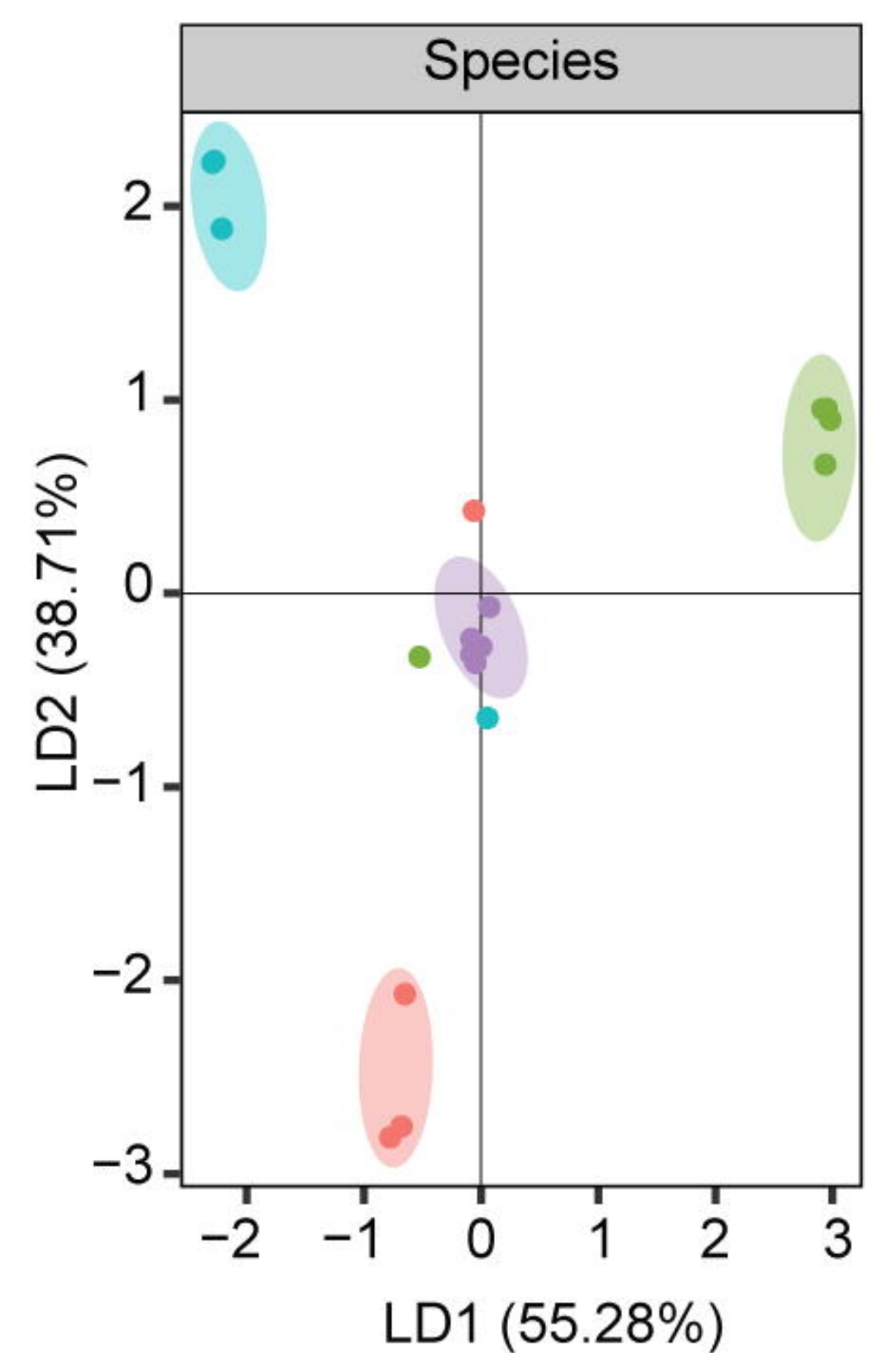


